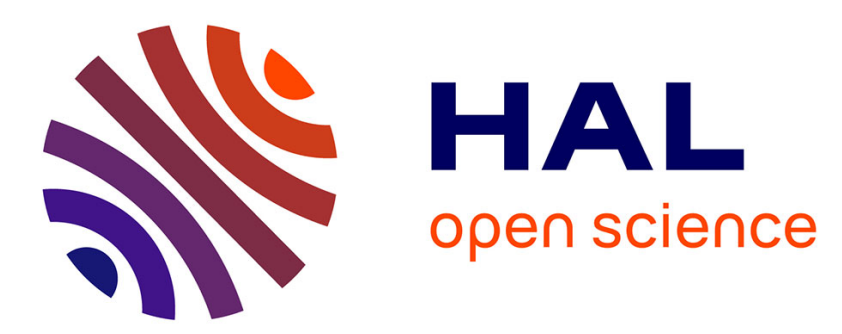

\title{
Fluid flow and effective conductivity calculations on numerical images of bentonite microstructure
}

\author{
Fatiha Bouchelaghem, R. Pusch
}

\section{To cite this version:}

Fatiha Bouchelaghem, R. Pusch. Fluid flow and effective conductivity calculations on numerical images of bentonite microstructure. Applied Clay Science, 2017, 144, pp.9-18. 10.1016/j.clay.2017.04.023 . hal-01520230

\section{HAL Id: hal-01520230 \\ https://hal.sorbonne-universite.fr/hal-01520230}

Submitted on 12 May 2017

HAL is a multi-disciplinary open access archive for the deposit and dissemination of scientific research documents, whether they are published or not. The documents may come from teaching and research institutions in France or abroad, or from public or private research centers.
L'archive ouverte pluridisciplinaire HAL, est destinée au dépôt et à la diffusion de documents scientifiques de niveau recherche, publiés ou non, émanant des établissements d'enseignement et de recherche français ou étrangers, des laboratoires publics ou privés. 


\title{
Fluid flow and effective conductivity calculations on numerical images of bentonite microstructure
}

\author{
F. Bouchelaghem ${ }^{\mathrm{a}, *}$, R. Pusch ${ }^{\mathrm{b}}$ \\ ${ }^{a}$ Sorbonne Universites, UPMC Univ Paris 06, CNRS, UMR 7190, Institut Jean Le Rond \\ d'Alembert, F-75005 Paris, France \\ ${ }^{b}$ Lulea University of Technology, Geotechnical Division, Porson, Lulea, Sweden
}

\begin{abstract}
Hydraulic conductivities of compacted water-saturated bentonite were computed based on the real microstructure. The Homogenization of Periodic Media approach employed fully acknowledges the heterogeneous and multiscale microstructure of clay, as well as locally varying physical flow properties. Consequently, three levels of description were considered : the microscopic level of clay particles, the mesoscopic level of clay aggregates, mineral grains and inter-aggregate porosity, and the macroscopic level of the sample subjected to fluid pressure gradients in the laboratory. Starting from the local description of fluid flow, the expression of the effective hydraulic conductivity tensor was derived. The soft and dense gels and the open voids may form a connected flow path or remain occluded. The local problems were solved on the microstructure obtained from a digitalized micrograph by image analysis. The contribution to macroscopic flow by the soft and dense gels was investigated in various configurations, and comparisons were made with hydraulic conductivity data for MX-80 bentonite.
\end{abstract}

Keywords: Hydraulic conductivity, Compacted water-saturated bentonite, Image analysis, Numerical Homogenization, Finite Element computations, Experimental comparison

\section{Introduction}

\subsection{Clay microstructure}

Smectites are characterized by a heterogeneous arrangement of nanometerscale lamellae stacked together to form clay particles, micrometer-scale grains, water and air which saturate the various pore spaces that appear on different scales (Jozja, 2003; Holzer et al., 2010; Keller et al., 2014; Pusch and Yong, 2006; Tessier et al., 1992). As a result, clay microstructure controls most of

\footnotetext{
${ }^{*}$ Corresponding author. Tel. (0033)147406816 Fax. (0033)0144278878

Email address: fatiha.bouchelaghem@upmc.fr (F. Bouchelaghem)
} 
their physical properties such as ion diffusion, swelling pressure and hydraulic conductivity. In water-saturated compacted bentonite, mesopores that contain free pore water may be distinguished from nanoscale pores that contain water molecules bound by surface forces. The multi-scale pore structure continuously changes during hydration, swelling and compaction processes, which complicates its characterization (Holzer et al., 2010; Tomioka et al., 2010). This explains the scarcity of microstructural data available to quantify the pore size distribution and pore connectivity in compacted bentonite. As pointed out in (Holzer et al., 2010; Keller et al., 2014), the proportions of free and bound porewater in compacted bentonite are the subject of ongoing discussions and investigations, and tomography techniques are still under development in order to quantify precisely the mesopore distribution in compacted swelling clays.

In (Pusch and Schomburg, 1999; Pusch, 2001) Transmission Electron Microscopy (TEM) micrographs of compacted and hydrated MX-80 bentonite displayed the formation of clay gels of variable density by linking of aggregates exfoliated from the expanding clay grains. Consequently, four main phases were distinguished: open voids which present no resistance to flow, clay gel-filled voids (designated as soft gels) which are characterized by a finite hydraulic conductivity, clay aggregates (designated as dense gels) and non-smectite grains which are assumed to be impermeable. Impervious zones and denser and softer gels were schematized by a grid of elementary cells with distinct hydraulic conductivities. The effective conductivity was then approximated by an analytical formula combining the individual cells in parallel or in series depending on their orientation with respect to the macroscopic flow direction. Tomioka et al. (2010) used Xray microtomography for examining the morphological evolution of compacted Na-montmorillonite before and after saturation. They concluded that the outer montmorillonite sheets are likely to swell and form a gel that occupies the intergranular voids, whereas the inner sheets are not affected by the water saturation process. Holzer et al. (2010) and Keller et al. (2014) investigated the intergranular pore space (mesopores) of compacted and hydrated MX-80 bentonite using high resolution 3D imaging with Focused Ion Beam nanotomography. They observed that clay particles' hydration in non-compacted bentonite (dry density $\rho_{\text {dry }}=0.39 \mathrm{~g} / \mathrm{cm}^{3}$ ) is associated with extensive exfoliation and dispersion of thin clay layers. At $\rho_{\text {dry }}=1.24-1.46 \mathrm{~g} / \mathrm{cm}^{3}$, clay aggregates located in the intergranular pore space displayed a honeycomb structure of stacked clay platelets filled with a colloidal clay gel of lower density. At higher densities, the small remaining fraction of mesopores were elongated in shape and no longer inter-connected, which led the authors to conclude that fluid flow and transport cannot take place through the mesopores alone. In compacted hydrated bentonites such as those intended for radioactive waste disposal, the isolated mesopores are connected only through the nanopores present within the clay matrix. As we will see in Section 3.1 the macroscopic fluid flow in the case of unconnected mesopores is then controlled by the conductivity of the clay matrix. 


\subsection{Multiscale modelling of fluid flow in geomaterials}

In recent studies (Adler et al., 1990; Al-Omari and Masad, 2004; Blunt et al., 2013; Masad et al., 2000) the effective permeability of various porous materials (natural sandstones, carbonate reservoirs, asphalt pavements, sands, glass beads) was determined by solving the local equations of fluid flow. The microstructure was obtained directly from the sample by image processing, or idealized by utilizing statistical characteristics. The computed flow fields displayed preferential paths depending on the size and connectivity of the pores, with whole areas of the pores relatively isolated from the flow. In the study by Blair et al. (1996), a two-point spatial correlation function obtained from image processing was employed to estimate several microstructural parameters (porosity, specific surface area, mean grain size, effective pore size), and combined with a Kozeny-Carman relation to estimate the intrinsic permeability of porous glasses and natural sandstones. Blunt et al. (2013) applied network modelling at larger scales to extract a topologically representative network consisting of pores and throats from the image, and the flow and transport properties were described semi-analytically. In (Adler et al., 1990; Al-Omari and Masad, 2004; Masad et al., 2000), the computed velocity field was driven by the pressure difference prescribed between the inlet and the outlet of the microstructure. Periodic boundary conditions were imposed for the velocity components, Darcy's law was written at the macroscopic scale and the effective permeability tensor was simply defined as the ratio between the average fluid velocity vector and the known pressure gradient. The previous studies concerned materials of intrinsic permeability higher than $10^{-12} \mathrm{~m}^{2}$, and did not investigate very low permeability materials such as compacted clays. Moreover, the pore space comprised only intergranular porosity with no nanoporosity such as encountered in clays.

Ichikawa et al. (1999, 2001, 2004a) and Wang et al. (2003) employed the Homogenization of Periodic Media to model fluid flow through clays. The microscopic and the macroscopic fluid flow equations were obtained from the Navier-Stokes equations using double scale asymptotic expansions, the two levels of description being related by the macroscopic permeability. Clay material was composed of lamellae of hydrated montmorillonite on the nanometric scale, while the mesoscopic domain consisted of clay particles and non-smectite grains. Water was distributed within the interlamellar and interparticle spaces, disregarding the mesopores. The computed effective permeability changed significantly with the void ratio, and comparison with experimental data (Pusch, 1994) confirmed the difficulties of assessing the permeability of very low permeability clays.

\subsection{Modelling approach and outline of the paper}

An experimental investigation on saturated bentonite (Pusch and Schomburg, 1999) has been employed in a numerical homogenization model in order to relate the macroscopic hydraulic conductivity with structural and microtextural properties. With respect to existing works on compacted bentonites (Bouchelaghem and Jozja, 2009a,b; Ichikawa et al., 1999, 2001, 2004a; Wang et 
al., 2003) which are all based on simplified microstructures (Poiseuille-like flow for lamellae of hydrated montmorillonite, symmetrical arrangements of spherical or cylindrical grains on the mesoscopic scale), the macroscopic permeability tensor is computed on real microstructures, while both mesopores and non-smectite grains are taken into account, leading to new effective permeability formulations. According to Baltean (1999) and Wodie (1992), the effective permeability will be obtained on the macroscopic scale by upscaling from the mesoscopic scale alone, consisting here of the arrangement of clay aggregates, inter-aggregate voids and non-smectite grains. As mentioned in Section 1.1, microstructural data regarding the intergranular space (Holzer et al., 2010; Keller et al., 2014; Pusch, 2001; Pusch and Yong, 2006) allow us to distinguish between dense and soft parts of the heterogeneous clay matrix, implying regions of low permeability and highly permeable channels. Accordingly, we assume that the soft gels always contribute to the macroscopic flow, while the dense gels may be impervious to fluid flow, completely open to flow, or characterized by an effective conductivity that can be determined from a separate upscaling approach.

The mesoscopic clay microstructure is described in Section 2.2, followed by the construction of a Finite Element mesh for numerical treatment in Section 2.3. Based on the description of effective fluid flow behaviour on the mesoscopic scale within clay aggregates (Section 2.4) and within inter-aggregate pores (Section 2.5 ), the method of asymptotic expansions is employed to derive the effective hydraulic conductivity tensor for unconnected mesopores (Section 3.1), for connected mesopores (Section 3.2), and when no mesopores are present (Section 3.3). Numerical simulations results and a comparison with experimental data on MX-80 bentonite are presented in Section 4. Finally, conclusions are drawn and future work is outlined in Section 5.

\section{Formulation of the problem on the mesoscopic scale}

\subsection{Materials and sample preparation}

MX-80 bentonite is a commercial smectite-rich clay, composed of numerous montomorillonite lamellae arranged in basic units or stacks (Pusch, 1999, 2001). The chemical constitution is as follows: $65-75 \%$ montmorillonite, 10$14 \%$ quartz, 5-9 \% feldspars, 2-4\% mica and chlorite, 3-5\% carbonates and chlorite, 1-3\% heavy minerals. The stacks are estimated to consist of 3-5 lamellae with equally oriented crystal axes in Na-saturated smectite, and about 10 lamellae if $\mathrm{Ca}$ is the dominant exchangeable cation (Pusch, 2001). Buffer clay blocks are prepared by compacting air-dry MX-80 clay powder at a compaction pressure of $100 \mathrm{MPa}$. The dry density of the grains is ca $1980 \mathrm{~kg} \mathrm{~m}^{-3}$ at 10 $\%$ by weight and the dry density of the powder mass poured in the form and slightly compacted is ca $1200 \mathrm{~kg} \mathrm{~m}^{-3}$. Transmission Electron Micrographs of very thin sections (about $310^{-2} \mu \mathrm{m}$ ) are obtained by acrylate impregnation of the clay under confinement, the sample preparation is detailed in Pusch (2001). 


\subsection{Microstructure}

The general description is based on observations of clay fabric that distinguish between two levels of arrangement: the particles and aggregation of particles (Holzer et al., 2010; Jozja, 2003; Pusch and Yong, 2006; Tessier et al., 1992).

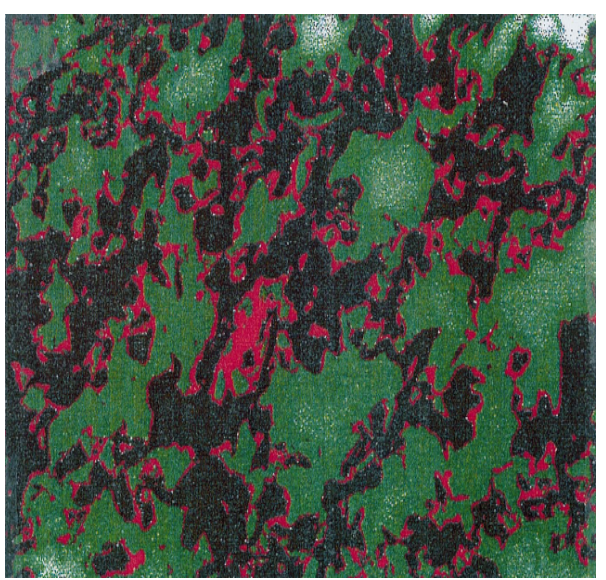

(a)

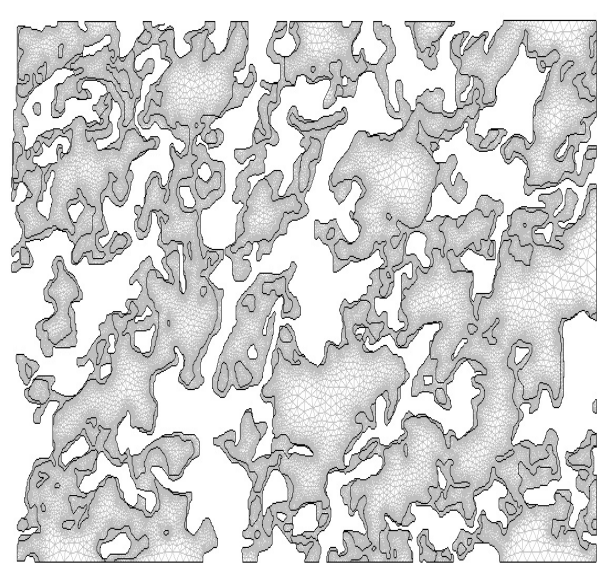

(b)

Figure 1: (a) : Digitalized micrograph of MX-80 clay, taken from (Pusch and Schomburg, 1999). (b) : Finite Element mesh of $1 / 4$ of the microstructure $\Omega$ for soft and dense gels, consisting of 271156 elements with 143862 mesh points.

The microscopic domain of clay particles, which is characterized by the length $l_{c}$, consists of stacked lamellae of montmorillonite, inter-layer water and lenticular pores (Bouchelaghem and Jozja, 2009a,b). Clay aggregates are represented by a periodical repetition of clay particles and inter-particle porosity. The mesoscopic domain is illustrated in Fig. 1a showing a TEM of MX-80 clay taken from Pusch (1999). The periodic microstructure $\Omega$, of characteristic length $l$, is composed of the porous matrix $\Omega_{c}$ of clay aggregates, water saturated inter-aggregate voids $\Omega_{f}$ which may be connected or not, and non-smectite grains $\Omega_{s}$ (in black in Fig. 1a). We will alternatively assume that the dense clay gels (in pink in Fig. 1a) are open to fluid flow and form a part of $\Omega_{f}$, or they will be considered as completely impervious, or they will be assimilated with a porous clay matrix $\Omega_{c}$ of low hydraulic conductivity. In a similar way, the soft clay gels (in green in Fig. 1a) will either be considered as open to fluid flow (as part of $\Omega_{f}$ ) or else be assimilated with a porous matrix of finite conductivity (contributing to $\Omega_{c}$ ). The mesoporosity $\phi_{m}$ is defined as the volume of inter-aggregate pores $\left|\Omega_{f}\right|$ divided by the total volume $|\Omega|$ :

$$
\phi_{m}=\frac{\left|\Omega_{f}\right|}{|\Omega|}=1-\frac{\left|\Omega_{c}+\Omega_{s}\right|}{|\Omega|}
$$

In the following, $\Gamma_{c f}$ and $\Gamma_{c s}$ respectively designate the interfaces between 
clay aggregates and inter-aggregate voids, and between aggregates and nonsmectite grains. $\Gamma_{f s}$ is the interface between mesopores and grains. Local periodicity is assumed for the microstructure and the physical properties, and Fig. 1b represents one quarter of the periodic cell $\Omega$. The periodicity assumption is equivalent to the assumption of local statistical homogeneity and ergodicity for all the physical variables in random media. The following conditions of separation of scales are taken to be verified (Bensoussan et al., 1978; SanchezPalencia, 1980; Rubinstein and Torquato, 1989; Torquato, 2005):

$$
\varepsilon^{\prime}=\frac{l_{c}}{L} \ll 1, \varepsilon=\frac{l}{L} \ll 1, \varepsilon^{\prime} \ll \varepsilon
$$

where $L$ designates the characteristic length of the macroscopic sample.

\subsection{Image analysis and construction of the FE mesh}

In order to create the geometry object to be meshed, the TEM micrograph in Fig. 1a is converted to a matrix format using the Image Processing Toolbox for Matlab (Matlab, 2010). In the resulting matrix of values ranging from 0 (black) to 255 (white), each level corresponds to a different density within the clay microstructure. A thresholding procedure based on a detailed exploration of the image is then performed, allowing to dissociate the three phases (soft gels and voids, dense gels, non-smectite grains) and resulting in a matrix with only three distinct values : 0 for the non-smectite grains, 125 for the dense gels and 255 for the soft gels and voids. The contours of the different phases are extracted and are imported into Comsol (Comsol, 2006) before being processed to obtain the surfaces occupied by the different phases. Fig. 1b represents the Finite Element unstructured mesh of the microstructure obtained by Delaunay triangulation. The relative fractions of the TEM picture are $41.6 \%$ for soft gels and voids, $18.2 \%$ for dense gels, and $40.2 \%$ for non-smectite grains. The soft gels and voids occupy $44.6 \%$ of the numerical microstructure, while the dense gels and grains represent respectively 20.2 and $35.2 \%$ of the microstructure. The difference between the relative proportions of the phases in the micrograph and the FE mesh is mainly due to the noise which is present in the processed TEM picture and cannot be completely eliminated by the thresholding procedure. Another source of error is the presence of very small inclusions of one phase within another phase, that do not contribute to the overall fluid flow and are neglected during the mesh generation. By varying the digitalized microstructure resolution, Al-Omari and Masad (2004) and Blunt et al. (2013) have observed that an approximately 1000-fold range from resolution to sample size may be necessary in order to obtain convergent permeability results. In our case, the high resolution employed, of the order of $10 \mathrm{~nm} /$ pixel in both the horizontal and the vertical directions, ensures a very good precision in capturing the actual microstructure and guarantees that the numerical permeabilities are convergent. Similar resolutions, comprised between 3.7 and $14.7 \mathrm{~nm} /$ pixel, have been employed to characterize the 2D and 3D mesopores distributions of MX-80 compacted hydrated bentonite (Holzer et al., 2010). Permeability computations 
similar to those depicted in Section 4 have also been performed on microstructures of increasing size, leading to convergent values for a microstructure size higher than two thirds of the microstructure illustrated in Fig. 1b.

\subsection{Mesoscopic description of the domain occupied by clay aggregates}

The derivation of the effective fluid flow behaviour through periodic media has been detailed in previous works for the case of constant fluid viscosity (Baltean et al., 2003; Baltean, 1999; Ichikawa et al., 1999, 2001; Levy, 1987; Wodie, 1992). For random media, the homogenization procedure is similar to the homogenization of periodic media, provided that the assumption of periodicity is replaced by the assumption of local statistical homogeneity and ergodicity (Rubinstein and Torquato, 1989; Torquato, 2005). The modelling approach proposed in Bouchelaghem and Jozja (2009a) in the case of variable viscosity can therefore be applied to random digitized clay microstructures such as the one illustrated in Fig. 1a.

In the inter-layer and inter-particle spaces $\Omega_{f}^{\prime}$, fluid flow, which is assumed to be periodical, laminar and stationary, is governed by Navier-Stokes equations in which inertia effects are neglected. Fluid viscosity $\mu=\mu\left(\mathbf{y}^{\prime}\right)$ is taken to vary locally in the microstructure, in order to account for the "ice sheet" effect of viscosity increase near the particle surface (Ichikawa et al., 1999, 2001, 2004a; Pusch and Yong, 2006). The mesoscopic description of flow in clay particles is obtained by performing an asymptotic analysis in terms of the parameter $\varepsilon^{\prime}$ (Bensoussan et al., 1978; Levy, 1987; Sanchez-Palencia, 1980; Rubinstein and Torquato, 1989), leading to (Bouchelaghem and Jozja, 2009a):

$$
\mathbf{v}^{0}=-\mathbf{k}_{j} \frac{\partial p^{0}}{\partial x_{j}}, p^{1}=-p_{j} \frac{\partial p^{0}}{\partial x_{j}}
$$

where $\mathbf{v}^{0}$ represents the first order approximation of fluid velocity, while $p^{0}=P$ (resp. $p^{1}$ ) is the first (resp. second) order approximation of fluid pressure, and $\mathbf{k}_{j}=\mathbf{k}_{j}\left(\mathbf{x}, \mathbf{y}^{\prime}\right)$ verify the following system of equations:

$$
\begin{array}{r}
\mu^{0} \Delta_{y^{\prime}} \mathbf{k}_{j}+\nabla_{y^{\prime}} \mu^{0} \cdot\left(\nabla_{y^{\prime}} \mathbf{k}_{j}+{ }^{T} \nabla_{y^{\prime}} \mathbf{k}_{j}\right)+\nabla_{y^{\prime}} p_{j}-\mathbf{e}_{i}=\mathbf{0}, \nabla_{y^{\prime}} \cdot \mathbf{k}_{j}=0 \text { in } \Omega_{f}^{\prime} \\
\mathbf{k}_{j}=\mathbf{0} \text { on } \Gamma_{s f}^{\prime}
\end{array}
$$

where $\mathbf{k}_{j}, p_{j}$ are $\Omega_{f}^{\prime}$-periodical. $\mathbf{e}_{i}$ is the unit vector along axis $y_{i}^{\prime}$. The term $\nabla_{y^{\prime}} \mu^{0} \cdot\left(\nabla_{y^{\prime}} \mathbf{k}_{j}+{ }^{T} \nabla_{y^{\prime}} \mathbf{k}_{j}\right)$ expresses the contribution of variable viscosity.

By applying the averaging operator to Eq. (3)a, we obtain a generalization of Darcy's law at the first order of approximation:

$$
\widetilde{\mathbf{v}}=\frac{1}{\left|\Omega^{\prime}\right|} \int_{\Omega_{f}^{\prime}} \mathbf{v} d V=\left(\varepsilon^{\prime}\right)^{2} \widetilde{\mathbf{v}}^{0}=-\left(\varepsilon^{\prime}\right)^{2} \widetilde{\mathbf{k}}_{j} \frac{\partial P}{\partial x_{j}}=-\mathbf{K} \nabla_{x} P
$$

In Eq. (5), $\widetilde{\mathbf{v}}=\left(\varepsilon^{\prime}\right)^{2} \widetilde{\mathbf{v}}^{0}$ represents the first order approximation of the fluid velocity within the porous space of clay particles, and $\mathbf{K}=\left(K_{i j}\right)$ is the hydraulic conductivity tensor of the porous clay matrix. 
The problem description within the domain $\Omega_{c}$ occupied by clay aggregates is completed by the continuity equation for the fluid phase, the non penetration condition on the interfaces $\Gamma_{c s}$ between clay aggregates and non-smectite grains ( $\mathbf{n}$ is the outward unit normal on the interface), as well as by $\Omega$-periodicity conditions:

$$
\begin{array}{r}
\nabla \cdot \widetilde{\mathbf{v}}=0 \text { in } \Omega_{c} \\
\widetilde{\mathbf{v}} \cdot \mathbf{n}=0 \text { on } \Gamma_{c s} \\
\widetilde{\mathbf{v}}, P \Omega-\text { periodical }
\end{array}
$$

\subsection{Mesoscopic description of the inter-aggregate voids}

In inter-aggregate pores, under slow flow conditions of incompressible free water, transient and inertia terms are assumed negligible. According to nuclear magnetic resonance tests cited in Pusch and Yong (2006), the viscosity of water in pore spaces smaller than about $5 \mathrm{~nm}$ is higher than in free water. Some reduction in fluidity may also occur in voids as wide as $10^{-2} \mu \mathrm{m}$, but for larger voids, the effect on porewater viscosity is estimated to be negligible. In the numerical simulations presented in Section 4, the smallest pore constrictions employed are around $0.05 \mu \mathrm{m}$. Consequently, fluid viscosity is assumed to be constant in mesopores and Navier-Stokes equations simplify to Stokes equations:

$$
\mu \Delta \mathbf{v}_{f}-\nabla p_{f}=0 \text { in } \Omega_{f}
$$

where $\mu, \mathbf{v}_{f}$, and $p_{f}$ respectively designate fluid viscosity, velocity and pressure within the mesopores. Mass balance is written as follows:

$$
\nabla \cdot \mathbf{v}_{f}=0 \text { in } \Omega_{f}
$$

Boundary conditions express fluid adherence on $\Gamma_{f s}$ and $\Omega$-periodicity:

$$
\begin{array}{r}
\mathbf{v}_{f}=\mathbf{0} \text { on } \Gamma_{f s} \\
\mathbf{v}_{f}, p_{f} \Omega-\text { periodical }
\end{array}
$$

We assume continuity of fluid pressures and normal velocities on both sides of $\Gamma_{c f}$ ( $\mathbf{n}$ designates the unit outward normal on $\left.\Gamma_{c f}\right)$ :

$$
\begin{array}{r}
p_{f}=P \text { on } \Gamma_{c f} \\
\mathbf{v}_{f} \cdot \mathbf{n}=\widetilde{\mathbf{v}} \cdot \mathbf{n} \text { on } \Gamma_{c f}
\end{array}
$$

\section{Determination of the macroscopic hydraulic conductivity}

Based on experimental evidence (Holzer et al., 2010; Keller et al., 2014; Pusch, 2001), the general description of a bentonite microstructure where clay gels of variable density partially fill the intergranular pore space has been retained. Three distinct formulations are obtained for the hydraulic conductivity at the macroscopic scale, depending on the distribution of the mesopores. 


\subsection{Effective hydraulic conductivity tensor with unconnected mesopores}

Depending on the initial fabric of the bentonite, connected flow paths of inter-aggregate pores may not exist (Bouchelaghem and Jozja, 2009b; Souli et al., 2008). In that case, according to the local description given in Sections 2.4 and 2.5, fluid flow in the clay aggregates is described by Darcy's law Eq. (5) and the continuity equation Eq. (6). In water-saturated unconnected mesopores, fluid flow is governed by Stokes equations Eq. (9) and the incompressibility condition, Eq. (10). At the boundary between both domains, we assume continuity of fluid pressure Eq. (13) and normal fluid velocity Eq. (14) while, at the boundaries of solid grains, Eqs. (7) and (11) are valid. To complete the problem description, Eqs. (8) and (12) express $\Omega$-periodicity. We must now consider that $\widetilde{\mathbf{v}}$ and $P$ depend on $\varepsilon$, in order to express the influence of mesopores and solid inclusions on fluid flow in the porous clay matrix.

As the unconnected voids $\Omega_{f}$ are surrounded by the porous matrix $\Omega_{c}$, fluid flow in the mesopores is a consequence of fluid flow in the clay matrix, which implies that fluid velocities and pressures will be of the same order of magnitude in both domains. The asymptotic expansions are defined accordingly, each physical quantity being a function of both the macroscopic position vector $\mathbf{x}$ and the microscopic position vector $\mathbf{y}$ describing the local scale. The results of homogenization of the system of Eqs. (5) to (14), constituting a generalization of the results by Wodie (1992), are detailed by Bouchelaghem and Jozja (2009a). We find that the local velocity field in the porous clay matrix is given by the following expression at the first order of approximation:

$$
\widetilde{v}_{i}^{0}=-K_{i j}^{0}\left(\delta_{j k}+\frac{\partial \Pi_{k}}{\partial y_{j}}\right) \frac{\partial p^{0}}{\partial x_{k}}
$$

Where the vector-valued function $\Pi_{k}$ does not depend on the macroscopic pressure gradient $p^{0}$ (defined everywhere in $\Omega$ ), which enables us to formulate the equivalent behaviour under the form of a generalized Darcy's law. The macroscopic fluid velocity $\left\langle\mathbf{V}^{0}\right\rangle$ is naturally defined as the volume average of the local fluid velocity fields in the clay aggregates $\Omega_{c}$ and the mesopores $\Omega_{f}$ :

$$
\left\langle\mathbf{V}^{0}\right\rangle=\frac{1}{|\Omega|}\left(\int_{\Omega_{c}} \widetilde{\mathbf{v}}^{0} d V+\int_{\Omega_{f}} \mathbf{v}_{f}^{0} d V\right)
$$

By use of the continuity equation, the adherence condition at the interface between the mesopores and the clay matrix, the periodicity condition and the divergence theorem, we obtain the final expression for the macroscopic velocity $\langle\mathbf{V}\rangle$ at the first order of approximation. It defines the effective hydraulic conductivity tensor $\mathbf{K}^{\mathrm{ncv}}$ for the case of unconnected mesopores:

$$
\begin{array}{r}
\langle\mathbf{V}\rangle=\left(\varepsilon^{\prime}\right)^{2}\left\langle\mathbf{V}^{0}\right\rangle=-\mathbf{K}^{\mathrm{ncv}} \nabla_{x} p^{0} \\
K_{i j}^{\mathrm{ncv}}=\frac{1}{|\Omega|} \int_{\Omega_{c}} K_{k l} \frac{\partial}{y_{k}}\left(y_{i}+\Pi_{i}\right) \frac{\partial}{y_{l}}\left(y_{j}+\Pi_{j}\right) d V
\end{array}
$$


In order to compute $\mathbf{K}^{\mathrm{ncv}}$ with Eq. (17)b from a volume averaging procedure, the following local problem for $\Pi_{k}(k=1,2)$ is solved by the Finite Element method (Comsol, 2006):

$$
\begin{array}{r}
\frac{\partial}{\partial y_{i}}\left(K_{i j} \frac{\partial \Pi_{k}}{\partial y_{j}}\right)=-\frac{\partial K_{i k}}{\partial y_{i}} \text { in } \Omega_{c} \\
\Pi_{k}=-y_{k} \text { on } \Gamma_{c f} \\
K_{i j} \frac{\partial \Pi_{k}}{\partial y_{j}} n_{i}=-K_{i k} n_{i} \text { on } \Gamma_{c s} \\
\Pi_{k}=0 \text { on } y_{k}=0, L_{k}^{e} \\
\frac{\partial \Pi_{k}}{\partial y_{j}} n_{j}=0 \text { on } y_{i}=0, L_{k}^{e}, i \neq k
\end{array}
$$

where $L_{k}^{e}(k=1,2)$ is the side length (along $\left.y_{k}\right)$ of the mesh in Fig. $1 b$.

\subsection{Effective hydraulic conductivity tensor with connected mesopores}

As in the case of unconnected mesopores, the mesoscopic description of fluid flow is given by the system of Eqs. (5) to (14). In connected mesopores, fluid velocity is now of order $O\left(\varepsilon^{2}\right)$, while according to Eq. (5), fluid velocity in the domain of clay aggregates is of order $O\left(\left(\varepsilon^{\prime}\right)^{2}\right)$ and can be neglected. Consequently, the continuity of fluid velocities on $\Gamma_{\varepsilon c f}$ will lead to a condition of null velocity for fluid flowing in the mesopores. The effective hydraulic conductivity tensor is obtained by upscaling of Stokes equations and neglecting fluid flow within clay aggregates, and the homogenization procedure leads to the local problem formulated in Section 2.4 in the particular case of constant viscosity:

$$
\mathbf{v}_{f}^{0}=-\mathbf{k}_{j}^{f} \frac{\partial p^{0}}{\partial x_{j}}
$$

where $p^{0}$ is the macroscopic pressure, and the local problem is written as:

$$
\begin{array}{r}
\mu \Delta_{y} \mathbf{k}_{l}^{f}+\nabla_{y} p_{l}-\delta_{i l} \mathbf{e}_{i}=\mathbf{0}, \nabla_{y} \cdot \mathbf{k}_{l}^{f}=0 \text { in } \Omega_{f} \\
\mathbf{k}_{l}^{f}=\mathbf{0} \text { on } \Gamma_{s f}, \mathbf{k}_{l}^{f} \cdot \mathbf{n}=0 \text { on } \Gamma_{c f} \\
p_{l}=0, \mathbf{k}_{l}^{f} \cdot \mathbf{t}=0 \text { on } y_{l}=0, L_{l}^{e} \\
\nabla_{y} p_{l} \cdot \mathbf{n}=0, \mathbf{k}_{l}^{f} \cdot \mathbf{e}_{j}=0 \text { on } y_{j}=0, L_{j}^{e}, j \neq l \\
\nabla_{y} \mathbf{k}_{l}^{f} \cdot \mathbf{n}=0 \text { on } y_{j}=0, L_{j}^{e}, \mathbf{j}=1,2
\end{array}
$$

$\mathbf{k}_{l}^{f}, p_{l}$ being $\Omega_{f}^{\prime}$-periodical. $\mathbf{e}_{i}$ is the unit vector along the local axis $y_{i}$.

Using Eq. (19) and defining the average fluid velocity $\langle\mathbf{V}\rangle$ as the volume average of the local velocity field over $\Omega_{f}$, we have the following expression for $\langle\mathbf{V}\rangle$ at the first order of approximation, defining the effective hydraulic 
conductivity $\mathbf{K}^{\mathrm{CV}}$ for connected mesopores as:

$$
\langle\mathbf{V}\rangle=\varepsilon^{2}\left\langle\mathbf{V}^{0}\right\rangle=\frac{\varepsilon^{2}}{|\Omega|} \int_{\Omega_{f}} \mathbf{v}_{f}^{0} d V=-\mathbf{K}^{\mathrm{cv}} \nabla_{x} p^{0}, K_{i j}^{\mathrm{cv}}=\frac{\varepsilon^{2}}{|\Omega|} \int_{\Omega_{f}} k_{i j}^{f} d V
$$

The local problem of Eqs. (20) to be solved for $\left(\mathbf{k}_{j}^{f}, p_{j}\right)$ is identical to the classical problem describing fluid flow of an incompressible Newtonian fluid under steady state conditions. The pressure gradient is taken equal to unity along the coordinate axis $x_{l}$ (of unit vector $\mathbf{e}_{l}$ ), and zero in the other directions:

$$
\frac{\partial p^{0}}{\partial x_{j}}=\delta_{j l}
$$

The effective hydraulic conductivity tensor $\mathbf{K}^{\mathrm{cV}}$ is then obtained through volumetric averaging on $\Omega_{f}$ according to Eqs. (21) and (22).

\subsection{Effective hydraulic conductivity with no mesopores}

In highly compacted clays the mesoporosity is negligible (Bouchelaghem and Jozja, 2009b; Holzer et al., 2010; Keller et al., 2014), and Eqs. (5) to (8) describe fluid flow in the clay aggregates. $\widetilde{\mathbf{v}}$ and $P$ depend on $\varepsilon$ in order to account for the influence of solid inclusions at the mesoscopic scale on fluid flow in the porous clay matrix. This problem has been studied by Baltean et al. (2003); Baltean (1999) and Wodie (1992). By introducing asymptotic expansions, and using the same approach as described in Section 3.2, we obtain the local fluid velocity as:

$$
\widetilde{v}_{i}^{0}=-K_{i j}\left(\delta_{j k}+\frac{\partial \Pi_{k}^{\prime}}{\partial y_{j}}\right) \frac{\partial p^{0}}{\partial x_{k}}
$$

The macroscopic fluid velocity is defined as the volume average of the local velocity field $\widetilde{\mathbf{v}}^{0}$, and from Eq. (23) we obtain at the first order of approximation:

$$
\langle\mathbf{V}\rangle=\left(\varepsilon^{\prime}\right)^{2}\left\langle\mathbf{V}^{0}\right\rangle=-\mathbf{K}^{\mathrm{nv}} \nabla p^{0}, K_{i j}^{\mathrm{nv}}=\frac{1}{|\Omega|} \int_{\Omega_{c}} K_{i k} \frac{\partial\left(y_{j}+\Pi_{j}\right)}{\partial y_{k}} d V
$$

Eq. (24)b defines the effective hydraulic conductivity tensor $\mathbf{K}^{\mathrm{nv}}$ in the particular case when there are no mesopores present in the microstructure, and the system to be solved for $\Pi_{k}^{\prime}$ takes the following form:

$$
\begin{array}{r}
\frac{\partial}{\partial y_{i}}\left(K_{i j} \frac{\partial \Pi_{k}}{\partial y_{j}}\right)=-\frac{\partial K_{i k}}{\partial y_{i}} \text { in } \Omega_{c} \\
K_{i j} \frac{\partial \Pi_{k}}{\partial y_{j}} n_{i}=-K_{i k} n_{i} \text { on } \Gamma_{c s} \\
\Pi_{k}=0 \text { on } y_{k}=0, \frac{L_{k}^{e}}{2} \\
\frac{\partial \Pi_{k}}{\partial y_{j}} n_{j}=0 \text { on } y_{i}=0, \frac{L_{i}^{e}}{2}, i \neq k
\end{array}
$$


Knowing $\Pi_{k}$, the effective hydraulic conductivity $\mathbf{K}^{\mathrm{nv}}$ is obtained by Eq. (24)b through volume averaging.

\section{Results and discussion}

Numerical computations have been performed in the case of isotropy of the clay matrix : $K_{i j}=K_{c} \delta_{i j}$, where $K_{c}$ is constant. The longitudinal permeability corresponds to the horizontal axis $\mathbf{x}$ while the transverse permeability is associated with the vertical axis $\mathbf{y}$ in the system of cartesian coordinates.

\subsection{Connected mesopores}

This situation is encountered for the determination of the transverse hydraulic conductivity, and either the connected mesopores correspond to the soft gels alone and the contribution of dense gels is neglected (Case a.), or the mesopores are associated with both the soft and dense gels (Case b.).

Case a. Soft gels.

The local problem of Eqs. (20) is solved in the domain occupied by the soft gels only, while the dense gels are assumed to be impermeable to flow. Fig. 2 illustrates the norm of the fluid velocity field computed within one quarter of the periodic cell. Fluid flow is highly localized and limited to the connected gel network at the left in the microstructure. The transverse intrinsic permeability value is equal to $K_{y y}^{\mathrm{CV}}=1.041 \times 10^{-17} \mathrm{~m}^{2}$. The range of corresponding hydraulic conductivity values, obtained from the classical relationship $K_{y y}^{h y d r}=\frac{\rho_{b u l k} g}{\mu} K_{y y}^{\mathrm{cV}}$, with $\rho_{\text {bulk }}=1300 \mathrm{~kg} / \mathrm{m}^{3}$ (Pusch and Schomburg, 1999) and $\mu$ confined between 1.8 and $4 \mathrm{mPa}$ s (Paumier, 2007), are listed in Table 1. In the horizontal direction there are no connected flow paths for the soft gels alone, therefore the effective longitudinal permeability cannot be computed using this approach, and will be obtained instead under the assumption of unconnected mesopores. The off-diagonal term $K_{x y}^{\mathrm{cV}}=K_{y x}^{\mathrm{cV}}=5.387 \times 10^{-18} \mathrm{~m}^{2}$, obtained from Eq. (21), is not negligible compared to the transverse permeability.

Case b. Soft and dense gels.

When all the gels are assumed to be open to fluid flow, the computed transverse effective permeability is equal to $K_{y y}^{\mathrm{cV}}=3.787 \times 10^{-16} \mathrm{~m}^{2}$. Using the same data as in Case a., the corresponding range of values for $K_{y y}^{h y d r}$ are listed in Table 1. The fluid velocity field is represented in Fig. 3, which shows connected flow paths as well. As in the simulations of Masad et al. (2000), the fluid flow is locally laminar but irregularly distributed within the void space. Narrow necks control the flow, while large portions of soft gels do not contribute to the overall fluid flow through the microstructure: fluid flows along preferential paths.

In the longitudinal direction there are no connected flow paths, the corresponding effective conductivity will be computed as below in Section 4.2. The off-diagonal component of the permeability tensor is obtained by integrating the horizontal velocity over the whole domain of soft and dense gels. From Eq. (21), we deduce $K_{y x}^{\mathrm{cv}}=1.0923 \times 10^{-16} \mathrm{~m}^{2}$. 


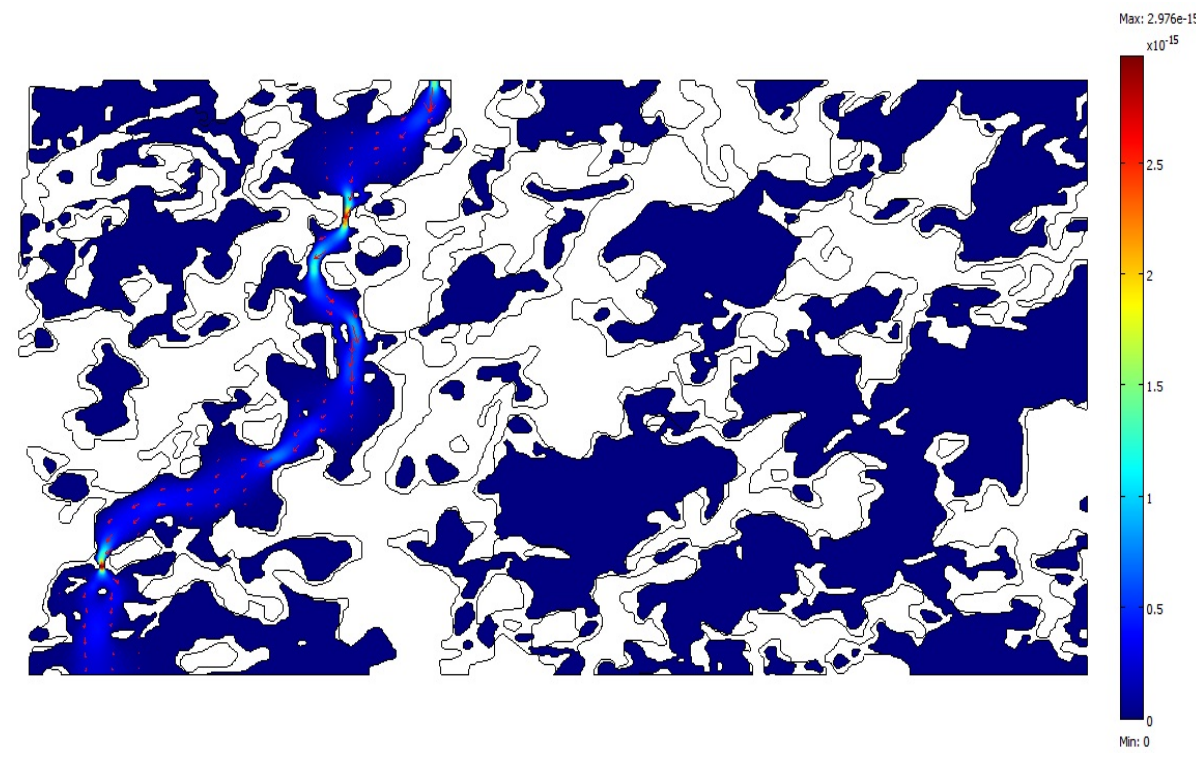

Figure 2: Connected mesopores. Fluid velocity field in Case a (soft gels only).

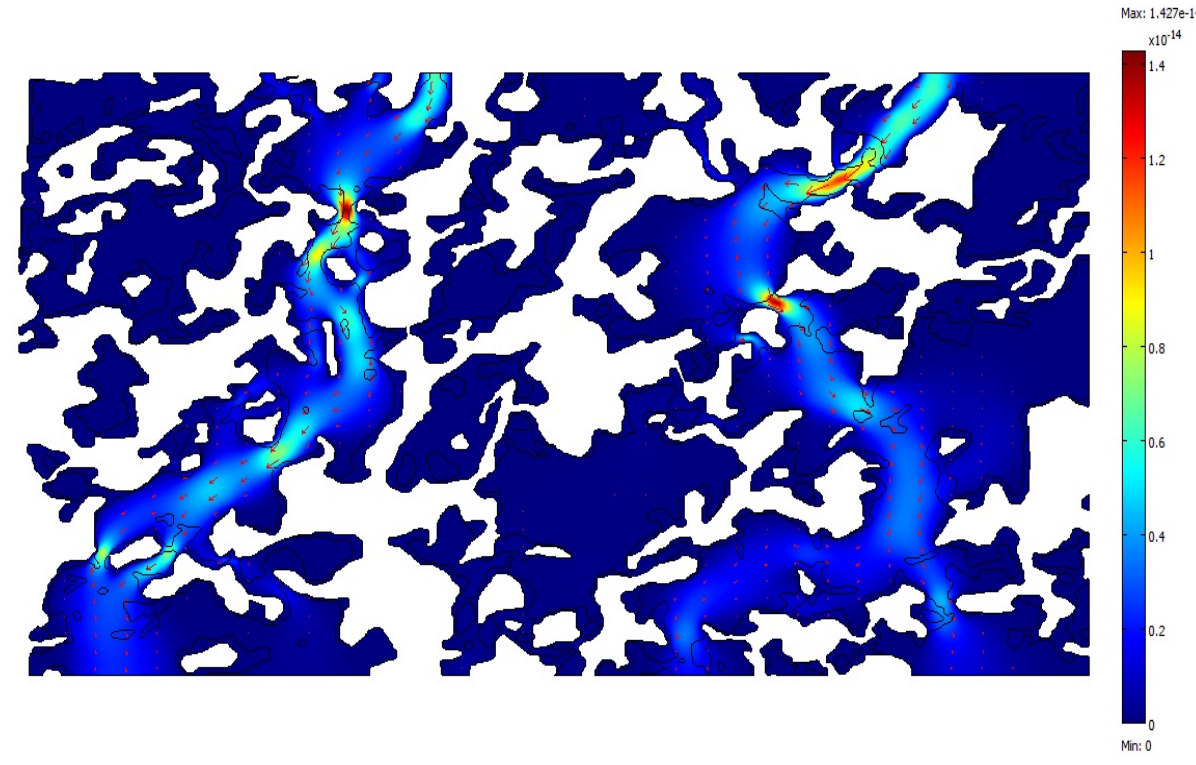

Figure 3: Connected mesopores. Fluid velocity field norm and vector in Case b (soft and dense gels). 


\subsection{Unconnected mesopores}

On the micrograph displayed in Fig. 1a, the soft gels alone do not form a continuous network throughout the whole microstructure. In that case, the hydraulic conductivity is obtained by solving the local system of Eqs. (19) in the domain $\Omega_{c}$ occupied by the dense gels of hydraulic conductivity $K_{c}$. Fig. 4 represents the distribution of fields $\Pi_{1}$ and $\Pi_{2}$. Dirichlet boundary condition on the interface between the soft and dense gels $\left(\Pi_{k}=-y_{k}\right.$ on $\left.\Gamma_{c f}, k=1,2\right)$ plays the role of a source term, (Bouchelaghem and Jozja, 2009a). The numerical intrinsic permeabilities are given in Table 1.

Table 1 Macroscopic hydraulic conductivities computed with unconnected mesopores and no mesopores.

\begin{tabular}{|l|l|l|}
\hline & $K_{\text {transverse }}$ & $K_{\text {longitudinal }}$ \\
\hline $\begin{array}{l}\text { Connected mesopores } \\
\text { Soft gels }\end{array}$ & $3.319 \times 10^{-11}-7.735 \times 10^{-11}$ & $\sim 0$ \\
\hline $\begin{array}{l}\text { Connected mesopores } \\
\text { Soft }+ \text { dense gels }\end{array}$ & $1.207 \times 10^{-9}-2.683 \times 10^{-9}$ & $\sim 0$ \\
\hline $\begin{array}{l}\text { Unconnected } \\
\text { mesoporess }\end{array}$ & $0.0950 K_{c}$ & $0.1095 K_{c}$ \\
\hline $\begin{array}{l}\text { No mesopores } \\
\text { Soft gels }\end{array}$ & $0.0215 K_{c}$ & $\sim 0$ \\
\hline $\begin{array}{l}\text { No mesopores } \\
\text { Soft + dense gels }\end{array}$ & $0.1294 K_{c}$ & $4.9667 \times 10^{-5} K_{c}$ \\
\hline
\end{tabular}

We can also compute the off-diagonal components of the permeability tensor from Eq. (17), $K_{x y}^{\mathrm{ncv}}=0.00197 K_{c}$, and $K_{y x}^{\mathrm{ncv}}=0.0020 K_{c}$, showing, as expected, that the effective permeability tensor is symmetric. After diagonalization, the principal values of the permeability tensor are given by: $K_{1}=0.1098 K c, K_{2}=0.0947 K c$, these results are very close to the permeabilities computed in the cartesian coordinate system, the principal directions being only slightly inclined (about $0.131 \mathrm{rad}$ ) with respect to the cartesian axes.

\subsection{No mesopores}

The permeabilities are obtained by solving the system of Eqs. (26) and the averaging defined in Eq. (24). We distinguish two cases, depending on whether the dense gels contribute to the overall fluid flow or not.

Case a. Soft gels.

The transverse and longitudinal permeability values are summarized in Table 1. Fig. 5 illustrates the local distribution of fields $\Pi_{1}$ and $\Pi_{2}$. As in the unconnected macrovoids case, Neumann condition (26)b on the interface between the clay gels and non-smectite grains $\left(\frac{\partial \Pi_{k}}{\partial y_{i}} n_{i}=-n_{k}\right.$ on $\left.\Gamma_{c s}, k=1,2\right)$ plays the role of a linear source of force.

Case b. Soft and dense gels.

The computed transverse and longitudinal permeabilities are listed in Table 1. Fig. 6 displays the distributions of $\Pi_{1}$ and $\Pi_{2}$ within the microstructure. As in Case a., the longitudinal permeability is negligible with respect to the transverse 


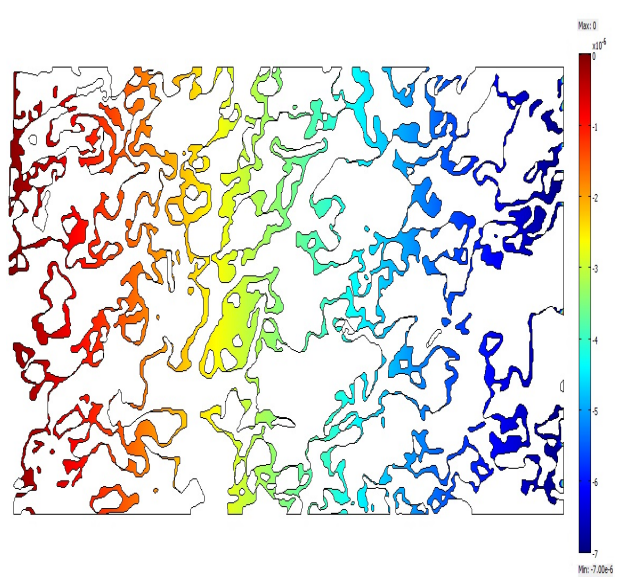

(a)

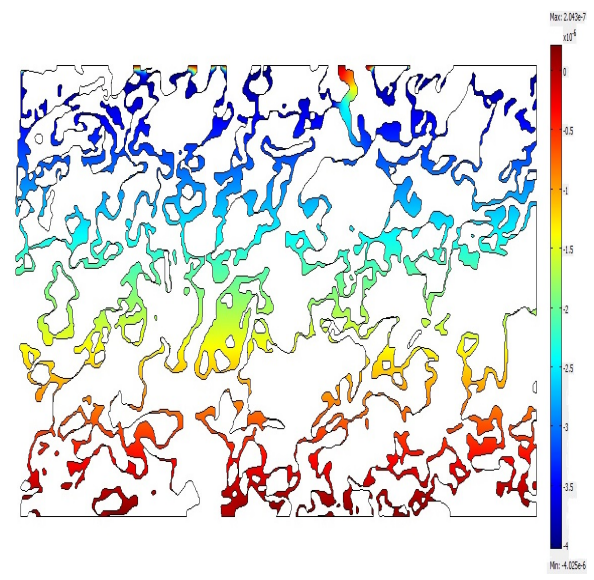

(b)

Figure 4: Unconnected mesopores. Distribution of fields $\Pi_{1}$ (a) and $\Pi_{2}$ (b) in the dense gel (porous matrix) domain.

permeability. The transverse permeability is of the same order of magnitude as the value obtained when soft gels are treated as unconnected mesopores.

\subsection{Discussion}

The permeability values computed for 3D microstructures are always higher than those obtained by a 2D analysis. According to Adler et al. (1990); Al-Omari and Masad (2004) and Masad et al. (2000), the discrepancy between 2D and 3D computations can be attributed to the inherent limitation of a $2 \mathrm{D}$ computation in capturing the real 3D flow path in a highly heterogeneous material such as sand or clay, as well as to the difference in specific surface area between the discretized contours of the pore space and the real microstructure. Therefore, $2 \mathrm{D}$ computations can be improved by increasing the resolution of the images in order to capture the real specific surface. As the surface area of the sand samples investigated by Masad et al. (2000) increases, the number of flow paths decreases and they are more tortuous as well, while narrower necks appear within the porous space leading to a ratio between the average 3D permeability and the average 2D permeability of 1.28 . Since in the present study there is no significant difference between the experimental and numerical surface areas, and as the surface area is notably higher for clays than for sands, we may reasonably assume that the $2 \mathrm{D}$ permeability is of the same order of magnitude as the $3 \mathrm{D}$ permeability.

Another aspect of interest is the anisotropy of the computed permeability values, which is measured by Masad et al. (2000) as the ratio $r_{k}$ of the horizontal permeability $k_{h}$ to the vertical permeability $k_{v}, r_{k}=\frac{k_{h}}{k_{v}}$. In their study, $r_{k}$ ranges from 1.1 for spherical glass beads to 1.67 for silica sand, and the overall 


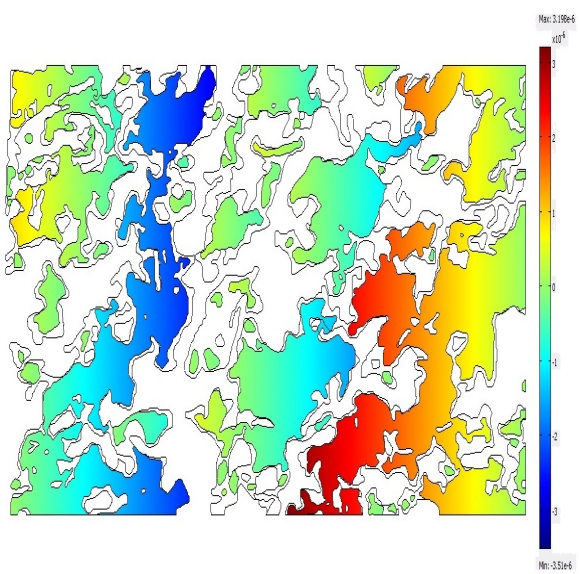

(a)

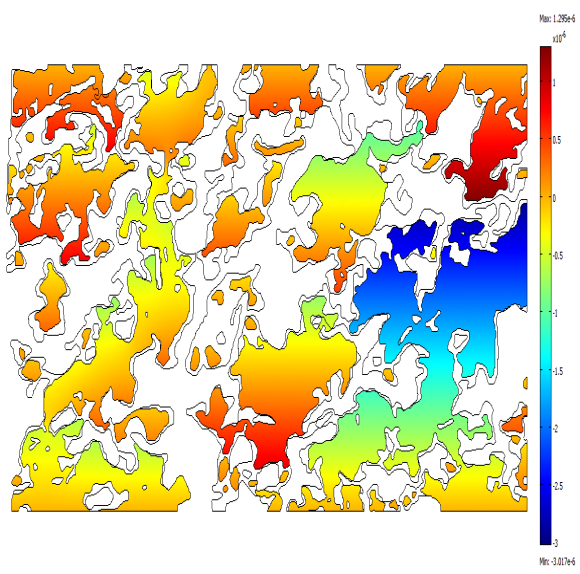

(b)

Figure 5: No mesopores. Iso-values of $\Pi_{1}(\mathrm{a})$ and $\Pi_{2}(\mathrm{~b})$ in the case of clay matrix limited to soft gels.

agreement between the 2D and 3D computations, as well as between the computations and the experimental values, increases with $r_{k}$. Masad et al. (2000) have shown that the permeability anisotropy is related to an increase in surface area and to the anisotropy of the porosity distribution in the samples. Our results are consistent with their findings. The anisotropy ratio for the case of unconnected intergranular voids is $r_{k}=0.8624$. In the connected mesopores as well as in the no-mesopores simulations, the permeability is highly anisotropic since the longitudinal permeability is negligible, implying that $r_{k} \simeq 0$.

\subsection{Comparison with experimental data}

$\mathrm{MX}$-80 in Ca-form.

For Wyoming clay in $C a$-form, the gel conductivity value $K_{\text {gel }}=7 \times 10^{-5}$ $\mathrm{m} / \mathrm{s}$ has been obtained by Pusch and Yong (2006) for $\rho_{\text {bulk }}=1570 \mathrm{~kg} / \mathrm{m}^{3}$. Using this value for $K_{c}$, we obtain in the unconnected mesopores simulations the results synthesized in Table 2. Results are also given for the simulations with no mesopores, with a permeable fraction limited either to soft gels alone, or attributed to both the soft and dense gels.

Table 2 Macroscopic hydraulic conductivities computed for $\mathrm{Ca}$-clay (All values are in $\mathrm{m} / \mathrm{s}$ ). 


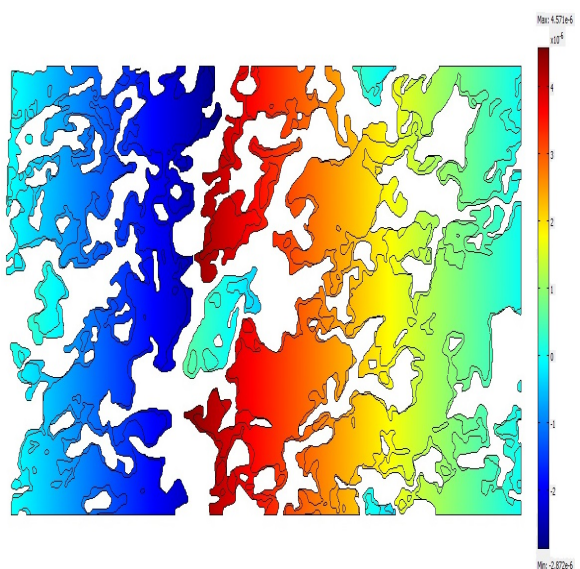

(a)

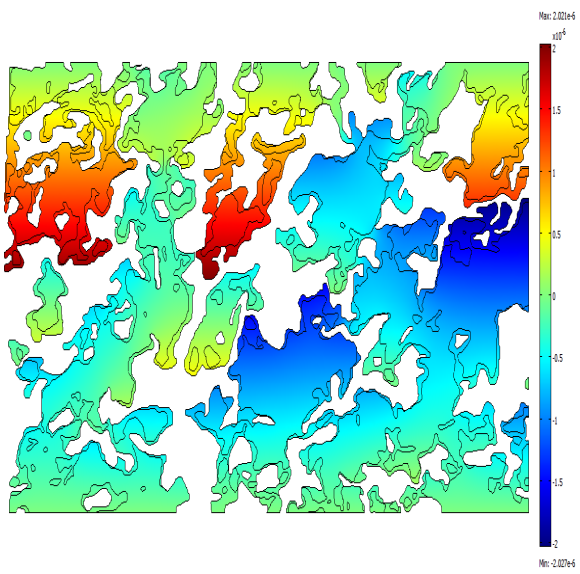

(b)

Figure 6: No mesopores. Iso-values of $\Pi_{1}(\mathrm{a})$ and $\Pi_{2}$ (b) in the case of soft and dense gels.

\begin{tabular}{|l|l|l|}
\hline & $K_{\text {transverse }}$ & $K_{\text {longitudinal }}$ \\
\hline $\begin{array}{l}\text { Unconnected } \\
\text { mesoporess }\end{array}$ & $6.65 \times 10^{-6}$ & $7.665 \times 10^{-6}$ \\
\hline $\begin{array}{l}\text { No mesopores } \\
\text { Soft gels }\end{array}$ & $1.505 \times 10^{-6}$ & $\sim 0$ \\
\hline $\begin{array}{l}\text { No mesopores } \\
\text { Soft }+ \text { dense gels }\end{array}$ & $9.058 \times 10^{-6}$ & $3.4767 \times 10^{-9}$ \\
\hline
\end{tabular}

Compared to the experimental bulk conductivity of $K_{\exp }^{C a}=2 \times 10^{-9} \mathrm{~m} / \mathrm{s}$, the model with connected mesopores and permeable soft and dense gels behaves well, and the no-mesopores model in the longitudinal direction gives interesting results as well. For the other models, the computed permeability is either several orders of magnitude higher than the experimental value or negligible. More values are required to draw a definite conclusion. Still, the calculations indicate that both the soft and dense clay gels contribute to flow for MX-80 in $\mathrm{Ca}$-form. $M X-80$ in $N a$-form.

For Wyoming clay in $N a$-form, the gel conductivity value $K_{\text {gel }}=2 \times 10^{-10}$ $\mathrm{m} / \mathrm{s}$ given in Pusch and Yong (2006) is four orders of magnitude lower than the Wyoming clay conductivity in $\mathrm{Na}$-form measured under similar compaction $\left(\rho_{\text {bulk }}=1570 \mathrm{~kg} / \mathrm{m}^{3}\right)$. Using this value for $K_{c}$, the results of the simulations are gathered in Table 3 .

Table 3 Macroscopic hydraulic conductivities computed for $N a$-clay (All values are in $\mathrm{m} / \mathrm{s})$. 


\begin{tabular}{|l|l|l|}
\hline & $K_{\text {transverse }}$ & $K_{\text {longitudinal }}$ \\
\hline $\begin{array}{l}\text { Unconnected } \\
\text { mesopores }\end{array}$ & $1.9 \times 10^{-11}$ & $2.19 \times 10^{-11}$ \\
\hline $\begin{array}{l}\text { No mesopores } \\
\text { Soft gels }\end{array}$ & $4.3 \times 10^{-12}$ & $\sim 0$ \\
\hline $\begin{array}{l}\text { No mesopores } \\
\text { Soft }+ \text { dense gels }\end{array}$ & $2.588 \times 10^{-11}$ & $\sim 0$ \\
\hline
\end{tabular}

The experimental conductivity measured in Pusch and Yong (2006) is equal to $K_{\mathrm{exp}}^{N a}=8 \times 10^{-11} \mathrm{~m} / \mathrm{s}$. The best agreement between the model and the experimental value is when the soft gels are assumed to make up the connected mesopores network. When both the soft and dense gels are included in the connected mesopores model, the computed permeability values are too high. The permeabilities computed using unconnected mesopores and no mesopores are slightly lower but of the same order of magnitude as the experimental data.

Using idealized microstructures, Bouchelaghem and Jozja (2009a) obtained an overall agreement between the experimental results of Pusch and Yong (2006) and the model with connected mesopores. However, the modelling with the mesopores filled with a permeable fraction of gels of finite conductivity must not be dismissed. Bouchelaghem and Jozja (2009b) have shown that for watersaturated Wyoming bentonite in $\mathrm{Mg}$ form, numerical conductivities using unconnected spherical mesopores in 3D microstructures compare very well with the conductivities measured before and after pollutant permeation.

\section{Conclusion}

In the present study, the effective hydraulic conductivity tensor was computed by solving the local Stokes equations of fluid flow through a $2 \mathrm{D}$ microstructure of a hydrated MX-80 bentonite. Starting from a digitized TEM micrograph that distinguishes between open voids, soft and dense gels and nonsmectite grains, different models were proposed which differ by the way the clay gels are treated. Those models have been developed in order to evaluate the contribution to the overall hydraulic conductivity of clay gels of varying density, and to assess the effect of mesopore connectivity. The different models were compared with existing experimental data available for MX-80 clay under $C a$ and $\mathrm{Na}$ form, showing that the description with connected mesopores does not necessarily lead to the highest values of hydraulic conductivity. More specifically, for clay under $\mathrm{Ca}$-form, the clay gel hydraulic conductivity is much higher than the bulk hydraulic conductivity measured, implying that the computed values of hydraulic conductivity with the unconnected mesopores model are too high. In that case, the best agreement with the experimental conductivity was obtained in the connected mesopores configuration, when both the soft and dense gels are open to fluid flow. For clay under $\mathrm{Na}$-form, the hydraulic conductivity computed with connected mesopores associated with soft gels alone was close to the experimental value. The latter results are consistent with the experimental observations by Pusch (2001) showing that in the $C a$-state the smectite 
stacks are thicker, the soft clay gels consist of bigger aggregates yielding larger open voids than in the $\mathrm{Na}$-state. However, for clay under $\mathrm{Na}$-form, the models with unconnected mesopores or with no mesopores resulted also in numerical hydraulic conductivities of the same order of magnitude as the experimental hydraulic conductivity.

A constant value was employed for the hydraulic conductivity of the clay gels which have been treated like a homogeneous phase. In fact, as observed by Keller et al. (2014) and Pusch (2001), the clay gels located in the cores of the honeycomb structure present a lower density and a lower mechanical stability than the clay aggregates composing the structure. The rheological behaviour of the clay gels has therefore to be modelled using a variable viscosity (power law, yield stress for fluid flow) (Birgersson et al., 2009). Additionally, a variable density and anisotropic behaviour would enable to characterize more precisely the effective conductivity tensor of clay gels depending on their density.

A perspective of this work, still under development, is to obtain a $3 \mathrm{D}$ reconstruction of a hydrated compacted bentonite from a $2 \mathrm{D}$ thin section such as the one employed in our numerical computations. This would allow to compute the effective conductivity as well as other transport properties on a microstructure that reflects the morphology and the long-range connectivity of the real porous network (Tahmasebi and Sahimi, 2012).

\section{References}

Adler, P.M., Jacquin, C.G., Quiblier, J.A., 1990. Flow in simulated porous media. International Journal of Multiphase Flow 16(4), 691-712.

Al-Omari, A., Masad, E., 2004. Three-dimensional simulation of fluid flow in X-ray CT images of porous media. International Journal for Numerical and Analytical Methods in Geomechanics 28, 1327-1360.

Baltean, D., 1999. Etude de la dispersion d'un contaminant passif dans les milieux multiporeux. Doctoral Thesis, University Pierre and Marie Curie.

Baltean, D., Levy, T., Balint, S., 2003. Diffusion-convection in a porous medium with impervious inclusions at low flow rates. Transport in Porous Media 51, 19-39.

Bensoussan, A., Lions, J.L., Papanicolaou, G., 1978. Asymptotic Analysis for Periodic Structures, North-Holland, Amsterdam.

Birgersson, M., Borgesson, L., Hedstrom, M., Karnland, O., Nilsson, U., 2009. Bentonite Erosion. Final Report from Clay Technology. SKB Report TR-0934. Swedish Nuclear Fuel and Waste Management Co., Stockholm, Sweden.

Blair, S.C., Berge, P.A., Berryman J.G., 1996. Using two-point correlation functions to characterize microgeometry and estimate permeabilities of sandstone and porous glass. Journal of Geophysical Research 101 (B9), 20359-20375. 
Blunt, M.J., Bijeljic, B., Dong, H., Gharbi, O., Iglauer, S., Mostaghimi, P., Paluszny, A., Pentland C., 2013. Pore-scale imaging and modelling. Advances in Water Resources 51, 197-216.

Bouchelaghem, F., Jozja, N., 2009a. Multi-scale study of permeability evolution of a bentonite clay owing to pollutant transport. Part I. Model derivation. Engineering Geology 108, 119-132.

Bouchelaghem, F., Jozja, N., 2009b. Multi-scale study of permeability evolution of a bentonite clay owing to pollutant transport. Part II. Application to an Mg-bentonite. Engineering Geology 108, 286-294.

Comsol Multiphysics 3.3, 1994-2006. Comsol Inc., Stockholm, Sweden.

Holzer, L., Munch, B., Rizzi, M., Wepf, R., Marshall, P., Graule T. 2010. 3Dmicrostructure analysis of hydrated bentonite with cryo-stabilized pore water. Applied Clay Science 47, 330-342.

Ichikawa, Y., Kawamura, K., Nakano, M., Kitayama, K., Kawamura, H., 1999. Unified molecular dynamics and homogenization analysis for bentonite behavior : current results and future possibilities. Engineering Geology 54, 21-31.

Ichikawa, Y., Kawamura, K., Nakano, M., Kitayama, K., Seiki, T., Theramast, N., 2001. Seepage and consolidation of bentonite saturated with pure or saltwater by the method of unified molecular dynamics and homogenization analysis, Engineering Geology 60, 127-138.

Ichikawa, Y., Kawamura, K., Theramast, N., Kitayama, K., 2004a. Secondary and tertial consolidation of bentonite clay : consolidation test, molecular dynamics simulation and multiscale homogenization analysis, Mechanics of Materials 36, 487-513.

Jozja, N., 2003. Etude de matériaux argileux albanais. Caracerisation multiéchelle d'une bentonite magnésienne. PhD thesis, University of Orleans. Available online at http://tel.archives-ouvertes.fr/tel-00003740/en/.

Keller, L.M., Seiphoori, A., Gasser, P., Lucas, F., Holzer, L., Ferrari, A., 2014. The pore structure of compacted and partly saturated MX-80 bentonite at different dry densities. Clays and clay minerals 62 (3), 174-187.

Lévy, T., 1987. in Homogenization Techniques for Composite Media, Lecture Notes in Physics, 272, Springer-Verlag, Berlin.

Masad, E., Muhunthan, B., Martys, N., 2000. Simulation of fluid flow and permeability in cohesionless soils. Water Resources Research 36(4), 851-864.

Matlab version 7.10.0, R2010a, The Mathworks Inc., Natick, Massachusetts.

Paumier, S., 2007. Facteurs determinant la rheologie du systeme argile-eau pour des suspensions de smectites, Doctoral Dissertation, Ecole Superieure d'Ingenieurs de Poitiers, 2007. 
Pusch, R., 1994. Waste Disposal in Rock, Elsevier.

Pusch, R., 1999. Microstructural evolution of buffers. Engineering Geology 54, $33-41$.

Pusch, R., Schomburg, J., 1999. Impact of microstructure on the hydraulic conductivity of undisturbed and artificially prepared smectitic clay. Engineering Geology 54, 167-172.

Pusch, R., 2001. The microstructure of MX-80 clay with respect to its bulk physical properties under different environmental conditions. Technical Report TR-01-08, Swedish Nuclear Fuel and Waste Management, Stockholm, Sweden.

Pusch, R., Yong, R., 2006. Microstructure of Smectite Clays and Engineering Performance. Taylor and Francis.

Rubinstein, J., Torquato, S., 1989. Flow in random porous media: Mathematical formulation, variational principles, and rigorous bounds. Journal of Fluid Mechanics 206, 25-46.

Sanchez-Palencia, E., 1980. Non Homogeneous Media and Vibration Theory, Lecture Notes in Physics 127, Springer-Verlag, Berlin.

Souli, H., Fleureau, J.M., Trabelsi Ayadi, M., Besnard, M., 2008. Physicochemical analysis of permeability changes in the presence of zinc. Geoderma 145, $1-7$.

Tahmasebi, P., Sahimi, M., 2012. Reconstruction of 3D porous media using a single thin section. Physical Review E 85, 066709.

Tessier, D., Lajudie, A., Petit, J.C., 1992. Relation between the macroscopic behaviour of clays and their microstructural properties. Appl. Geochem. 1, $151-161$

Tomioka, S., Kozaki, T., Takamatsu, H., Noda, N., Nisiyama, S., Kozai, N., Suzuki, S., and Sato, S., 2010. Analysis of microstructural images of dry and water-saturated compacted bentonite samples observed with X-ray micro CT. Applied Clay Science 47, 65-71.

Torquato, S., 2005. Random heterogeneous materials. Microstructure and macroscopic properties, Interdisciplinary Applied Mathematics, Springer.

Wang, J.G., Leung, C.F., Chow, Y.K., 2003. Numerical solutions for flow in porous media. Int. J. for Numerical and Analytical Methods in Geomechanics $27,565-583$.

Wodie, J.C., 1992. Contribution a l'etude des milieux poreux par la methode de l'homogeneisation. PhD thesis, University Pierre and Marie Curie. 